\title{
EFFECTS OF RASTER ORIENTATION, INFILL RATE AND INFILL PATTERN ON THE MECHANICAL PROPERTIES OF 3D PRINTED MATERIALS
}

\author{
DUDESCU Cristian \\ professor, Faculty of Mechanical Engineering/Department of Mechanical Engineering, Technical \\ University of Cluj-Napoca, Romania,Mircea.Dudescu@rezi.utcluj.ro \\ RACZ Laszlo \\ Ph.D. student, Faculty of Mechanical Engineering/Department of Mechanical Engineering, Technical \\ University of Cluj-Napoca, Romania, sraczlaszlo@yahoo.com
}

\begin{abstract}
Three-dimensional printing is an additive manufacturing process that allows rapid design and manufacture of complex component based on computer-aided design models. Compared with some conventional manufacturing processes, additive manufacturing part properties can depend on structural and process parameters rather than purely on material properties. The objectives of the paper are to evaluate the tensile properties of $3 D$ printed components produced using a commercial 3D printer by performing standard tensile tests and to assess the influence of the technological parameters upon mechanical proprieties of printed specimens, considering different printing directions, infill rates and infill patterns. The influence of raster angles is tested through the designed specimens with different transverse plane, they are printed by placing in different angle, including $0^{\circ}, 30^{\circ}, 45^{\circ}$ and $90^{\circ}$. Specimens with an infill rate varying from $20 \%$ to $100 \%$ and six different infill patterns has been tested.
\end{abstract}

Key words: 3D printed materials, infill rate, infill patterns, tensile proprieties

\section{Introduction}

Three-dimensional (3D) printing is an additive manufacturing (AM) process that allows rapid design and manufacture of complex component based on computer-aided design models. The advent of open source, low cost 3D printers has led in the last years to a significant proliferation of this technology. Most low-cost commercial 3D printers utilize Fused Deposition Modeling (FDM) as the manufacturing process. FDM forms a 3D geometry by assembling individual layers of extruded thermoplastic filament. The manufacturing process is useful for rapidly producing prototypes but can be also used to produce functional components. FDM is especially promising for the fabrication of low volume products such as replacement parts for complex systems.

Fused deposition modeling (FDM) is a layered manufacturing technology that produces parts with complex geometries by the layering of extruded materials, such as acrylonitrile butadiene styrene (ABS) plastic or polyetherimide, the process consists in the deposition of filaments of the material at the semi-molten state. The partially melted filament is extruded though a heated nozzle within a temperature controlled build environment and is deposited in the form of a prescribed two-dimensional (x-y) layer pattern onto the partially constructed part. The material is extruded and laid in tracks at a molten state and the newly deposited material fuses with adjacent material that has already been deposited. After an entire layer is deposited, the build platform moves downward along the $\mathrm{z}$-axis by an increment equal to the filament height (layer thickness) and the next layer is deposited on top of it [1].

The mechanical properties of the layered components should meet the service loading and operational requirements and must be comparable with parts produced by traditional manufacturing techniques. Compared with the conventional manufacturing processes, additive manufacturing part properties can depend on structural and process parameters rather than purely on material properties. This is also the main disadvantage of utilizing FDM printed parts for functional components.

Due to this process effects as delamination of the component layers or materials anisotropy can occur. Additionally, printed components typically have lower elastic properties than injection molded components of the same thermoplastics, thus the designers cannot rely on values from static material databases, the material selection becoming a complex process. 
To determine if 3D printed materials can be used for functional components, the mechanical properties need to be determined [2-4] and is also important to predict not only the strength, but also the stiffness and how they relate to process parameters.

The main production parameters affecting the mechanical behavior of FDM manufactured components that have been identified and investigated can be summarized as follows [5]:

- Orientation (building direction): Part build orientation refers to the inclination of part in a build platform with respect to $\mathrm{X}, \mathrm{Y}, \mathrm{Z}$ axis. $\mathrm{X}$ and $\mathrm{Y}$-axis are considered parallel to build platform and $\mathrm{Z}$-axis is along the direction of part build.

- Bead width: It is the width of the filament deposited by the FDM nozzle (common ranges from $0.3 \mathrm{~mm}$ to $1 \mathrm{~mm}[6])$.

- Layer thickness: Thickness of the deposited bead and therefore of any single layer. It depends upon the type of nozzle used and is usually one half of the bead width and.

- Raster angle: It is a direction of raster relative to the $\mathrm{x}$-axis of build table.

- Part raster width (raster width): Width of raster pattern used to fill interior regions of part curves.

- Air gap (raster to raster gap): It is the gap between two adjacent raster's on same layer. The default value is usually zero, meaning that the beads touch each other [6]. A positive gap means that a gap is present between adjacent raster's, or a negative gap, implying that the bead tracks are overlapped. For many slicing software that drive the 3D printers it can be set only the infill rate, representing the density of the printed pattern. Therefore, even some specimens were printed with a setting of $100 \%$ infill, the actual positive or negative air gaps should be determined or estimated. This affects the part as air gap has been shown to be an important contributing factor to tensile strength $[7,8]$.

- Number of contours: the filament is initially deposited along the component edge, the inner part of the component is after that filled by inclined rasters upon the completion of the first layer and is repeated for the generation of all the other layers.

Mechanical test methods such as tension, compression, bending and impact are used to test the influences of the manufacturing parameters. The influences of parameters on mechanical properties are presented in the literature, thus Dawoud et al. [9] investigate the effect of processing technique on the mechanical behavior of virgin ABS, specifically different combination of raster angles and air gap in each printing layer of FDM components. Results show that an adequate selection of FDM parameters can reach mechanical properties comparable to those of injection molded parts in both static and dynamic loading modes. Ahn et al. [6] characterizes the properties of ABS parts. Using a Design of Experiment (DOE) approach, the process parameters of FDM, such as raster orientation, air gap, bead width, color, and model temperature were examined. Tensile strengths and compressive strengths of directionally fabricated specimens were measured and compared with injection molded ABS P400 material. The researcher presents in [10] an analytic model to predict the tensile strength of FDM parts and a predicted anisotropic failure model of the tensile strength as a function of raster angle by Tsai-Wu criterion. Sood et al. [11] established a strength model of FDM build parts for mechanical property prediction and parameters setup optimization purpose with varying inputs of building orientation, layer thickness, raster angle, raster width and air gap using response surface methodology. The effects generated by the FDM production parameters on the tensile strength and on the stiffness of the generated components, by analytical and experimental studies is presented by Croccolo et al. [5]. For this purpose, an analytical model was developed, which can predict the strength and the stiffness properties based on the number of contours deposited around the component edge and on the setting of the raster pattern parameters (dimensions, number of contours, raster angle). Rodriguez et al. [7] introduces a strategy for optimizing the design of FD-ABS components for stiffness and strength under a given set of loading conditions. A mathematical model of the structural system was linked to an approximate minimization algorithm to find the settings of select manufacturing parameters, which optimize the mechanical performance of the component. Tymrak et al. [12] demonstrated the equivalent tensile strength of the products produced by RepRap 3D printer and the commercial ones by different raster angles and layer 
heights. Montero et al. [13] experimentally characterized anisotropic properties of FDM produced parts by examining five printing process parameters, among which raster orientation and air gap had significant effects on tensile strength as compared to raster width, filament color and processing temperature. A negative air gap would increase both tensile strength and stiffness.

The first objective of this paper is to evaluate the tensile properties of 3D printed components produced using a commercial desktop 3D printer by performing conventional tensile tests. The second objective is to assess the influence of the technological parameters upon mechanical proprieties of printed specimens, considering different printing orientations, infill rate and infill patterns. The term "infill" refers to the structure that is printed inside an object. It is extruded in a designated percentage and pattern, which is set in the slicing software. The printing orientation is defined as the angle between the principal axis of the specimen and one axis of the printing platform.

\section{Materials and methods}

\subsection{Materials and printing parameters}

To start with FDM printing process, a geometric solid model is designed on CAD software and converted to a stereolithography (STL) formatted file. It is then imported into slicing software to be horizontally sliced into thin layers with two-dimensional contours information, by which the tool path of the process plan is generated to control the FDM machine hardware. FDM samples were produced using a Wanhao Duplicator i3 machine of $200 \times 200 \times 180 \mathrm{~mm}$ print volume. STL files were generated using Solid Works 2016 CAD software and further sliced into machine readable g-code using Simplify3D program version 3.1.0. The printing parameters used to manufacture the test specimens are presented in Table 1 .

Table 1. Printing parameters.

\section{FDM parameters.}

\begin{tabular}{ll}
\hline Parameter & Value \\
\hline Nozzle diameter & $0.75 \mathrm{~mm}$ \\
Primary layer height & $0.2 \mathrm{~mm}$ \\
No. of contours & 1 \\
Printing speed & $30 \mathrm{~mm} / \mathrm{s}$ \\
Nozzle temperature & $250^{\circ} \mathrm{C} \pm 5$ \\
Bed temperature & $100{ }^{\circ} \mathrm{C}$ \\
\hline
\end{tabular}

Test specimen print parameters.

\begin{tabular}{ll}
\hline Print parameters & \\
\hline Layer height (mm) & 0.2 \\
Infill percentage (\%) & $20-100 \%$ \\
Infill orinetation (degrees) & $0-30-45-90$ \\
Number of infill layers & 12 \\
Shell thickness & 0.4 \\
Number of shells & 3 \\
Nunber of floor layers & 4 \\
Number of ceiling layers & 4 \\
Number of solid layers & 8 \\
Total nr of layers & 20 \\
\hline
\end{tabular}

The material used for specimen's preparation was acrylonitrile butadiene styrene ABS filament (Plasty Mladeč, Czech Republic), a common used material for FDM processed parts. Its mechanical properties, according to the producer, are: tensile modulus $\mathrm{E}_{\mathrm{f}}=2140 \mathrm{MPa}$, tensile stress $\sigma_{\mathrm{f}}=43 \mathrm{MPa}$ and tensile strain $\varepsilon_{\mathrm{f}}=2,7 \%$.

The geometric model of 3D printing specimens is realized according to ISO 527-2-2012 (International standard, plastics Determination of tensile properties Part 2: Test conditions for molding and extrusion plastics) as shown in Figure 1. Uniaxial tensile tests are carried out to investigate the mechanical property of the 3D printed material. The specimens are tested in a universal testing machine type INSTRON 3366, $10 \mathrm{kN}$ capacity. The loading speed of this machine is $1 \mathrm{~mm} / \mathrm{min}$ and the test stops 
once the specimens broken. A uniaxial extensometer was used to measure the tensile strain. Stress calculation is done based on apparent cross-section of the specimen that do not account for the void area.

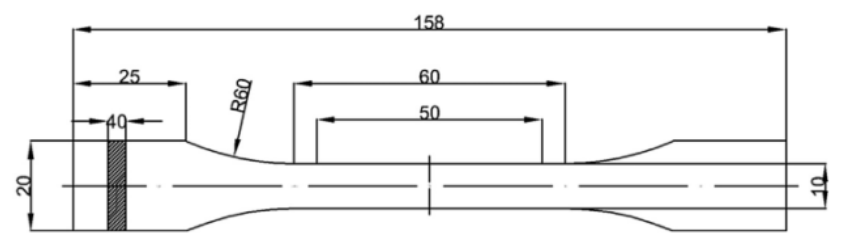

Figure 1: Dimensions of the tensile specimen

Three different types of specimens have been manufactured by varying the following parameters:

a) Infill rate. The infill rate states how much material there are in the printed pattern and represents the density of the pattern. For this study the values of $20 \%, 40 \%, 60 \%, 80 \%$ and $100 \%$ were considered. The exact air gap between extruded filament raster cannot be specified, the parts can be created solid or hollow by adjusting the percent infill, with $100 \%$ being completely solid.

b) Infill pattern. When using any infill percentage, a pattern is used to create a strong and durable structure inside the print. There are several different infill pattern options, each with advantages and tradeoffs between print time, material usage or strength of the obtained part. 3D printing software usually provides infill pattern selections for the users. For example, Simplify3D provides users with six infill pattern options, including Fast Honeycomb, Full Honeycomb, Wiggle, Triangular, Grid and Rectilinear infill patterns. For this paper were printed specimens with $100 \%$ infill and following patterns (Figure 2), rectilinear $0^{\circ}$ and $90^{\circ}$, grid $0^{\circ}-90^{\circ}$ and $45^{\circ}-45^{\circ}$, fast honeycomb, full honeycomb, triangular $\left(60^{\circ}\right)$ and wiggle.

c) Raster orientation. The raster angle $\alpha$ is defined as the angle between the principal axis of the specimen and the axis $\mathrm{X}$ of the printing platform. The specimens are printed by placing in different angle $(\alpha)$, including $0^{\circ}, 30^{\circ}, 45^{\circ}$ and $90^{\circ}$ as shown in Figure 3.

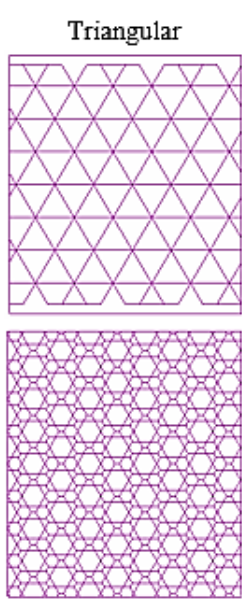

Full honeycomb



Fast honeycomb

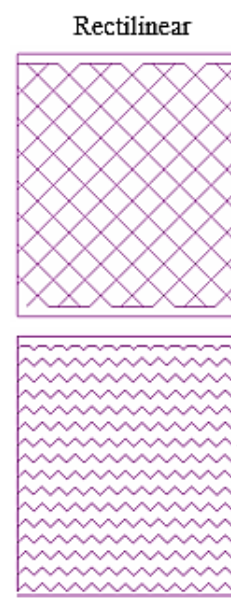

Wiggle

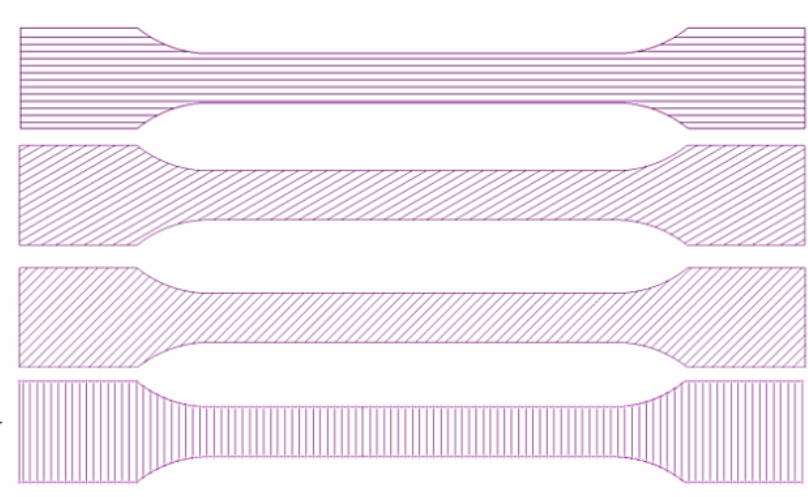

Printing platform

Figure 2: Infill patterns

To determine the mechanical properties of 3-D printed specimens and the variability in these properties when different user-controlled printing and slicing parameters are used, this study looked at the relationship between infill rate, infill pattern and raster orientation to tensile strength, strain at tensile strength, and Young's modulus. A complete set of 5 specimens of each of the combinations of above variables was printed and subjected to the tensile test. 
ACTA UIVERSITATIS CIBINIENSIS - TECHNICAL SERIES

Vol. LXIX 2017

\subsection{Influence of the infill rate}

Influence of the infill rate was studied on specimens filled with rectilinear $0^{\circ}$ orientation of the pattern with respect to the tensile direction. In Figure 4 is presented the variation of the Young's modulus, tensile stress, and tensile strain at maximum stress with the infill rate for the apparent cross-section.



a)



b)

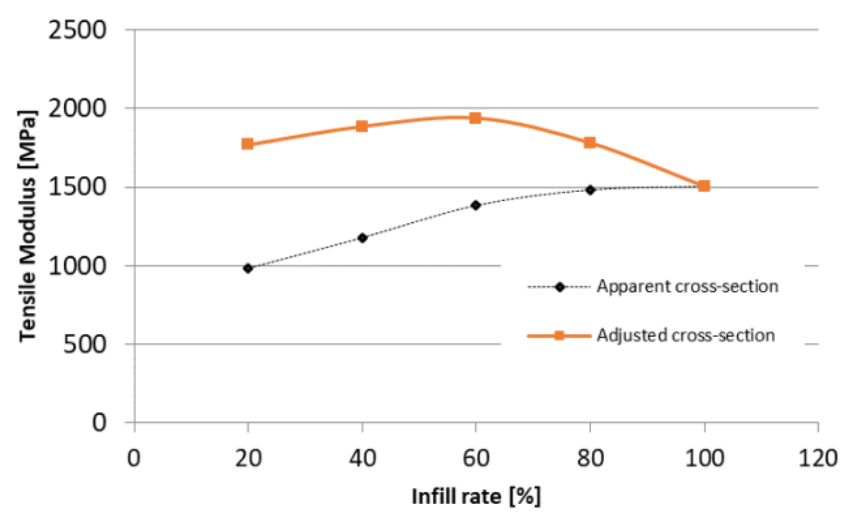

c)

Figure 4: Variation with the infill rate: a) tensile stress, b) tensile strain at maximum stress and

\section{c) Young's modulus}

As expected, the specimen strength increases with infill rate, from 16.1 MPa at $20 \%$ to $28.9 \mathrm{MPa}$ at $100 \%$, the evolution is not linear. An interesting result of the experimental investigations was obtained for the elongation at maximum stress that is around $2.2 \%$ excepting the value for $100 \%$ infill that is around $2.7 \%$, the value of ABS filament elongation. The apparent E-modulus has also in increase with the infill percentage from $982 \mathrm{MPa}$ at $20 \%$ to $1503 \mathrm{MPa}$ at $100 \%$, the increase per percentage point of infill also changes non-linearly, the higher the infill rates the smaller the increase (Figure 4c). The E-modulus behavior is due to the approximate formula to calculate the real cross-sectional area.

Because the specimen, excepting the $100 \%$ infill rate, is porous, the E-modulus can be calculated considering apparent cross section (ignoring the void inside the part) or the calculation can be adjusted by multiplying the cross-section area by the infill rate. In this case the adjusted E-modulus can be obtain similarly as with the rule of mixtures by multiplying the apparent modulus with the (1-Infill_rate).

$$
E_{\text {adjusted }}=(1-\text { Infill_rate }) E_{\text {apparen }}
$$

The adjusted E-modulus should have a more constant behavior around of the ABS filament value.

Even some specimens were printed with a setting of $100 \%$ infill, the actual positive or negative air gaps should be determined or estimated more precisely. Infill rates in the printer specification is referring only to the interior part of the specimen and do not consider the number of contours deposited 
around the component edge. The number of contours and their pattern doesn't change with the infill rate, for smaller infill rates, the outer contour has a significant area contribution that reflects in the E-modulus values.

\subsection{Influence of the infill pattern}

Influence of the infill pattern was studied on printed specimens with $100 \%$ infill and following patterns: rectilinear $0^{\circ}$ and $90^{\circ}$, grid $0^{\circ}-90^{\circ}$ and $45^{\circ}-45^{\circ}$, fast honeycomb, full honeycomb, triangular $\left(60^{\circ}\right)$ and wiggle. In Figure 5 is presented the variation of the Young's modulus, tensile stress and tensile strain at maximum stress with the infill pattern, considering apparent and calculated cross-section. Increase of the tensile strength can be noticed for $0^{\circ}, 90^{\circ}$ and wiggle patterns.

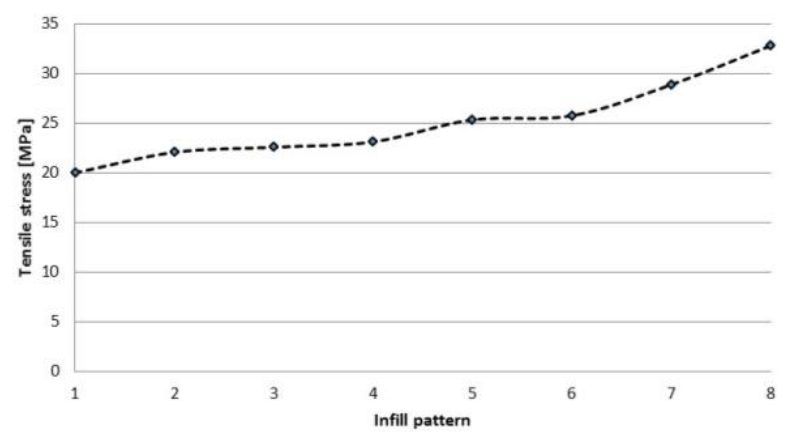

a)

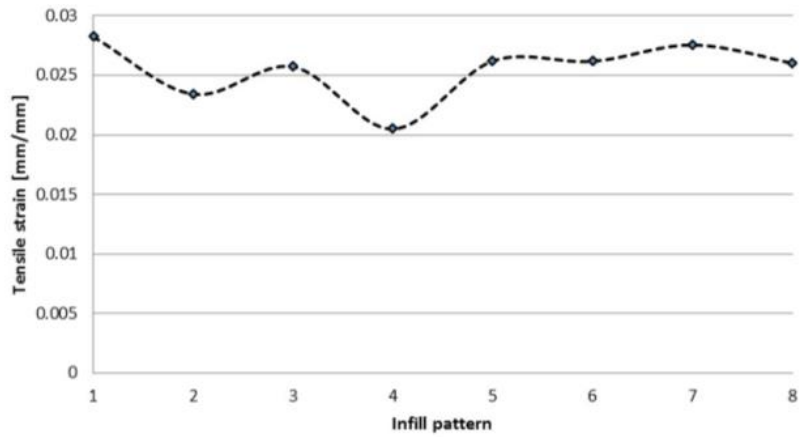

b)

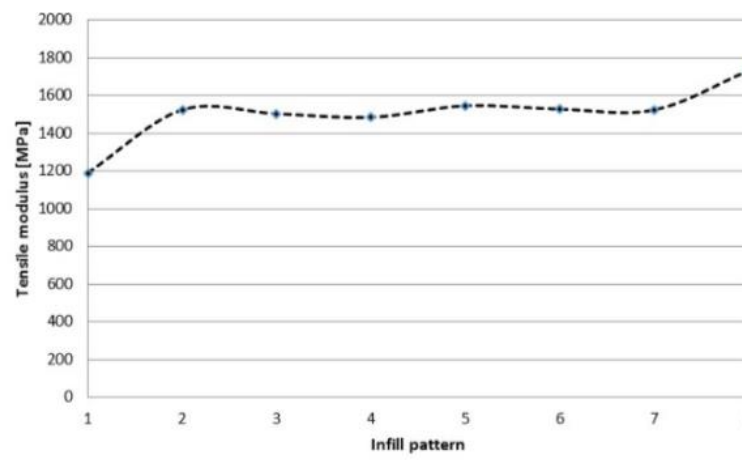

1) Full Honeycomb

2) 0_90_degree

3) Triangular

4) Fast Honeycomb

5) $45-45$ degree

6) $90 \_$degree

7) 0_degree

8) Wiggle

c)

Figure 5: Variation with the infill pattern: a) tensile stress, b) tensile strain at maximum stress c) Young's modulus

Triangular, hexagonal, grid $0^{\circ}-90^{\circ}$ and $45^{\circ}-45^{\circ}$ are all comparable in terms of strength and E-modulus. Elongation at break is between $2 \%$ and $2.8 \%$ with the smallest value for the fast honeycomb pattern.

\subsection{Influence of the raster orientation}

Specimens with $100 \%$ infill rate, rectilinear orientation of the pattern were printed by placing them in different angle about the axis $X$ of the printing platform (Figure 2), including $0^{\circ}, 30^{\circ}, 45^{\circ}$ and $90^{\circ}$. In Figure 6 is presented the variation of the tensile stress, tensile strain at maximum stress and Young's modulus with the raster orientation, considering apparent cross-section. The apparent tensile modulus have a more constant value around the value of $1500 \mathrm{MPa}$. 
ACTA UIVERSITATIS CIBINIENSIS - TECHNICAL SERIES

Vol. LXIX 2017

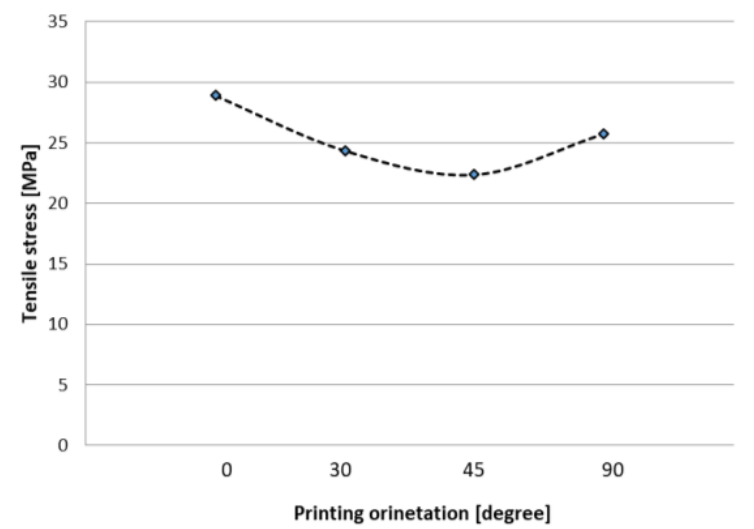

a)

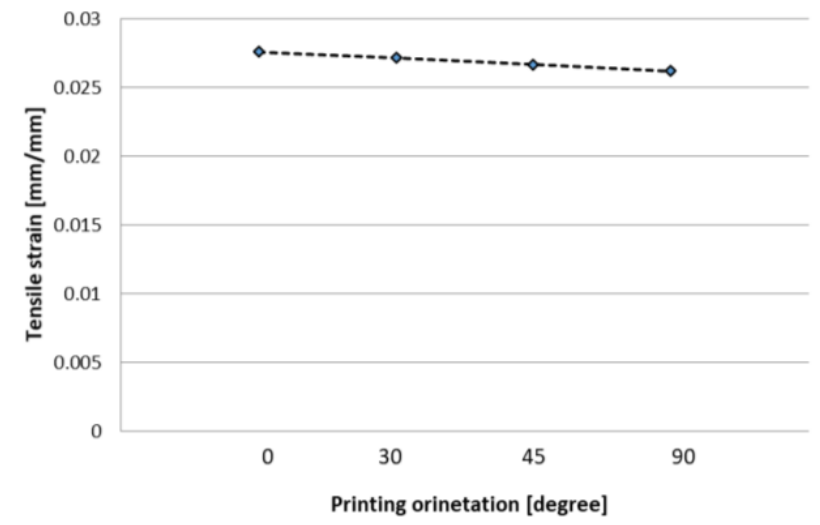

b)



c)

Printing orientation [degree]

Figure 7: Variation with the raster orientation: a) tensile stress, b) tensile strain at maximum stress and c) Young's modulus

\section{Conclusions}

The paper presents the measured values of tensile properties of 3D printed specimens produced using a desktop 3D printer. The influence of the technological parameters upon mechanical proprieties of printed specimens, considering different infill rates, infill patterns and printing orientations have been analyzed. For this study the infill rates of $20 \%, 40 \%, 60 \%, 80 \%$ and $100 \%$ were considered. The exact air gap between extruded filament raster cannot be specified, the parts can be created solid or hollow by adjusting the percent infill, with $100 \%$ being theoretically completely solid. Infill pattern influence has been studied for printed specimens with $100 \%$ infill and rectilinear $0^{\circ}$ and $90^{\circ}$, grid $0^{\circ}-90^{\circ}$ and $45^{\circ}-45^{\circ}$, fast honeycomb, full honeycomb, triangular $\left(60^{\circ}\right)$ and wiggle patterns. To study the raster orientation specimens with $100 \%$ infill rate, rectilinear orientation of the pattern was printed by placing them in different angle about the axis $X$ of the printing platform $\left(0^{\circ}, 30^{\circ}, 45^{\circ}\right.$ and $\left.90^{\circ}\right)$.

The mechanical properties of ABS specimens fabricated by fused deposition modelling display are significantly influenced not only by the infill rates as expected, but also about the printed pattern of different layers and their orientation. Even some specimens were printed with a setting of $100 \%$ infill, the actual positive or negative air gaps should be determined or estimated more accurate. The effect of the void geometry on the local stresses and strains will affect the macroscale mechanical behavior of the material. The apparent E-modulus has an increase with the infill percentage, the increase per percentage point of infill changes non-linearly, the higher the infill rates the smaller the increase. Adjusted E-modulus that consider the void density can be obtain similarly as with the rule of mixtures 


\section{ACTA UIVERSITATIS CIBINIENSIS - TECHNICAL SERIES}

Vol. LXIX 2017

and should have a more constant behavior around of the ABS filament value. The difference can be explained due to the approximate formula to calculate the real cross-sectional area that do not consider the number of contours deposited around the component edge. Tension tests indicate that the ultimate strengths are the largest for wiggle, $90^{\circ}$, and $0^{\circ}$ patterns in descending order. Orienting the raster along the printing direction lead to the largest tensile strength.

Results are useful to choose future analytical or computational models of FDM strength or stiffness as a function of printing patterns, void density and raster orientation. Different void density, pattern, orientations and their combination can be employed in producing parts that fulfill a required stiffness or strength. A local variation of this parameters can lead to an optimized structure that do not presume geometrical changes, just adjustments of the printing parameters.

\section{References}

1. Ziemian, C., M. Sharma, and S. Ziemian, Anisotropic mechanical properties of ABS parts fabricated by fused deposition modelling, in Mechanical engineering. 2012, InTech.

2. Melenka, G.W., et al., Evaluation and prediction of the tensile properties of continuous fiber-reinforced $3 D$ printed structures. Composite Structures, 2016. 153: p. 866-875.

3. Zou, R., et al., Isotropic and anisotropic elasticity and yielding of $3 D$ printed material. Composites Part B: Engineering, 2016. 99: p. 506-513.

4. Liljenhjerte, J., P. Upadhyaya, and S. Kumar, Hyperelastic strain measurements and constitutive parameters identification of $3 d$ printed soft polymers by image processing. Additive Manufacturing, 2016. 11: p. 40-48.

5. Croccolo, D., M. De Agostinis, and G. Olmi, Experimental characterization and analytical modelling of the mechanical behaviour of fused deposition processed parts made of ABS-M30. Computational Materials Science, 2013. 79: p. 506-518.

6. Ahn, S.-H., et al., Anisotropic material properties of fused deposition modeling ABS. Rapid prototyping journal, 2002. 8(4): p. 248-257.

7. $\quad$ Rodríguez, J.F., J.P. Thomas, and J.E. Renaud, Design of Fused-Deposition ABS Components for Stiffness and Strength. Journal of Mechanical Design, 2003. 125(3): p. 545-551.

8. Li, L., et al., Composite modeling and analysis for fabrication of FDM prototypes with locally controlled properties. Journal of manufacturing processes, 2002. 4(2): p. 129-141.

9. Dawoud, M., I. Taha, and S.J. Ebeid, Mechanical behaviour of ABS: An experimental study using FDM and injection moulding techniques. Journal of Manufacturing Processes, 2016. 21: p. 39-45.

10. Ahn, S.H., et al., Anisotropic Tensile Failure Model of Rapid Prototyping Parts - Fused Deposition Modeling (FDM). International Journal of Modern Physics B, 2003. 17(08n09): p. 1510-1516.

11. Sood, A.K., R.K. Ohdar, and S.S. Mahapatra, Parametric appraisal of mechanical property of fused deposition modelling processed parts. Materials \& Design, 2010. 31(1): p. 287-295.

12. Tymrak, B.M., M. Kreiger, and J.M. Pearce, Mechanical properties of components fabricated with opensource 3-D printers under realistic environmental conditions. Materials \& Design, 2014. 58: p. 242-246.

13. Montero, M., et al., Material characterization of fused deposition modeling (FDM) ABS by designed experiments. Society of Manufacturing Engineers, 2001. 10(13552540210441166). 